\title{
Photoemission from tin and lead cluster anions
}

\author{
G. Ganteför, M. Gausa, K.H. Meiwes-Broer, and H.O. Lutz \\ Fakultät für Physik, Universität Bielefeld, D-4800 Bielefeld 1, Federal Republic of Germany
}

\begin{abstract}
Photoelectrons from mass-identified jet-cooled tin and lead cluster anions $\left(\mathrm{Sn}_{n}^{-}, \mathrm{Pb}_{n}^{-}\right)$are detached by ultraviolet laser light $(h v=3.68 \mathrm{eV})$. The photoelectron energy spectra give the detachment energies of ground state cluster anions (electron affinities) as well as excitation energies of neutral clusters in the geometry of the anions. The energy spectra for $\mathrm{Sn}_{n}^{-}$are dominated by flat thresholds with an $n$-dependence similar to that of other group IV clusters. In contrast, for $\mathrm{Pb}_{n}^{-}$we find pronounced narrow lines close to threshold, generally followed by a $0.3-1.4 \mathrm{eV}$ gap which indicates closed-shell behaviour of $\mathrm{Pb}_{n}^{-}$for nearly all $n$.
\end{abstract}

PACS: $36.40 .+\mathrm{d} ; 33.60 . \mathrm{Cv} ; 79.60 .-\mathrm{i}$

\section{Introduction}

The physics of unsupported clusters has overcome the phase where mainly mass spectra and their-often speculative-interpretations were of main interest. With the development of intense ion and anion sources it is now possible to perform chemical or spectroscopic investigations on mass selected isolated clusters in a beam $[1,2]$. When dealing with metal clusters classical spectroscopic methods like flame or absorption spectroscopy, laser-induced fluorescence or resonant twophoton ionization are mostly sentenced to fail since in general electronic excitation energies are redistributed into vibrational degrees of freedom before light emission would occur or before a second photon can ionize the cluster. This rapid energy redistribution, as well as the low densities of free mass-selected cluster beams, have reduced much of the general instrumentarium of atomic, molecular and surface physics in its significance for cluster research. One technique which has recently been shown to remain a powerful tool is photoelectron spectroscopy (PES). Within a short time a large amount of photoelectron data of mainly metal or group IV clusters have become available (see, e.g., [3-6]). Although theory is lacking behind the experimental progress a number of physical properties can already be extracted from such PES data. In this paper
PE-spectra of tin and lead clusters will be presented; although the elements $\mathrm{Sn}$ and $\mathrm{Pb}$ are chemically similar, PES reveals the different nature of these group IV clusters.

\section{Experimental}

The experimental set-up has been discussed elsewhere $[5,6]$. In short, metal cluster anions are produced in a laser vaporization source without an additional ionization agent. After cooling in a (pulsed) supersonic jet the anions are mass-separated in a time-of-flight (TOF) mass spectrometer collinearly to the source direction. Under ultrahigh vacuum conditions fixedfrequency light of an ultraviolet laser detaches electrons from mass-identified anion bunches. The electron energies are analyzed by recording their flight times in a magnetic-bottle TOF electron spectrometer [7] perpendicular to the cluster beam direction. The energy resolution $\Delta \mathrm{E} / \mathrm{E}$ depends on a variety of experimental parameters, in particular the magnetic field configuration, the ionization volume and the anion's velocity (see [5]). In the spectra presented here $\frac{\Delta E}{E} \approx 0.05$ was achieved. Monomer anion photoelectron spectra serve to calibrate the electron spectrometer. 


\section{Results and discussion}

The cluster ion beam composition is studied by TOF mass analysis yielding broad, uniform intensity distributions for (positively and negatively) charged metal clusters $[5,6,8]$. Figure la gives as example the mass spectrum of $\mathrm{Sn}_{n}^{-}$where no intensity anomalies appear. Such structureless spectra can be produced for all metals we studied so far; we conclude that the source operates at aggregation conditions where the cluster ion growth is not determined by differences in cluster binding energies. A change of critical source parameters (e.g., detuning of the laser and source pulse overlap) can lead to more structured spectra. Figure $1 \mathrm{~b}$ gives as example the mass spectrum of $\mathrm{Pb}_{n}^{-}$where $\mathrm{Pb}_{7}^{-}$ and $\mathrm{Pb}_{10}^{-}$appear with enhanced intensity. In addition, an intensity step is observed at $\mathrm{Pb}_{15}^{-}$. Further detuning will lead to an enhancement of these features and to an overall decrease of cluster ion intensity. The appearance of irregularities might originate from cluster growth where the partial pressure of the He seeding gas during aggregation is too low for an effective transport of the heat of condensation. In this case
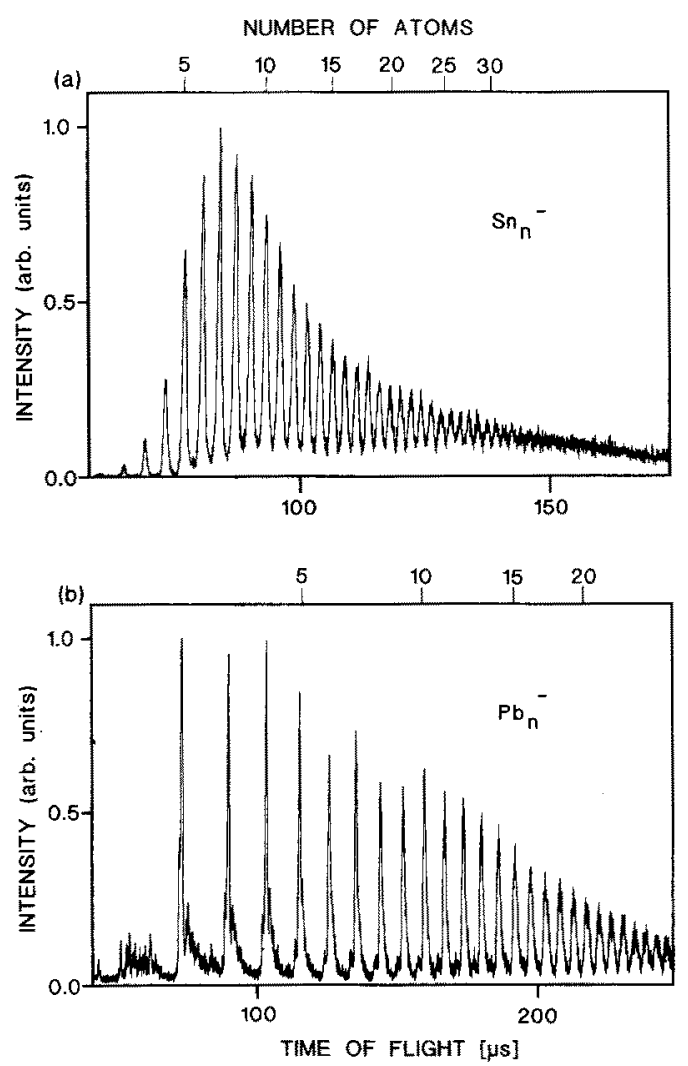

Fig. 1a and $\mathbf{b}$. Time-of-fight mass spectra of jet-cooled $\mathrm{Sn}_{n}^{-}$(upper trace) and $\mathrm{Pb}_{n}^{-}$(lower trace). The anions are produced in a laser vaporization source (without additional ionization) and analyzed collinearly to the source direction. For $\mathrm{Pb}_{n}^{-}$the source parameters are slightly detuned in order to produce "magic numbers" condensation heats the clusters and can lead to a decomposition before sufficient stabilization in collisions with He would occur. "Magic numbers" result, reflecting differences in cluster binding energies. It is interesting to note that the pronounced lines in $\mathrm{Pb}_{n}^{-}$ spectrum at $n=7,10,15$ also appear at the same positions in $\mathrm{Pb}_{n}^{+}$spectra after electron impact ionization [9] or photoionization [10]. Therefore, simple electron counting rules or the jellium model cannot explain these numbers. Other source operation conditions may produce other pronounced lines, e.g., $\mathrm{Pb}_{12}^{-}$. Negatively charged 13-atom clusters, in contrast to positive ones, do not appear with enhanced intensity at all. This finding sheds some doubt on the interpretation based on positive cluster abundances according to which $\mathrm{Pb}_{13}$ is built in an icosahedral structure. Instead, the close-packing model should be extended by detailed quantum-chemical considerations in which the influence of electronic symmetries is included.

To investigate the electronic structure of the cluster anions, photoelectrons have been detached by pulsed laser light $(h v=3.68 \mathrm{eV})$. Resulting electron flight times are converted numerically into electron binding energies. Figure $2 a, b$ gives such energy distribution curves (EDC) of photoemitted electrons for tin and lead cluster anions. All cluster anions detach at photon energies of $<3 \mathrm{eV}$. For each cluster a characteristic EDC is obtained, starting with a more or less pronounced threshold. Such spectra reflect the distribution of transitions from the anionic ground states into the ground states of the neutralized clusters (in the geometry of the anions) or into their excited states: Distinct narrow lines near threshold (see Table 1) are interpreted as vertical transitions into the respective neutral electronic ground state (electron affinities); peaks at the high energy sides of the first lines belong to excitations into higher electronic neutral states, i.e. the energetic positions of the electronic states are revealed directly by photoelectron spectroscopy. In contrast to optical spectroscopy, however, a larger amount of states is accessible since the only important "selection rule" is the change of the spin multiplicity by one.

Most of the tin cluster EDCs do not show narrow peaks near threshold. Therefore, PE-thresholds (as marked by bars in Fig. 2, see also Table 1) are rough estimates of tin electron affinities. A similar threshold behaviour is observed also for a number of other metal clusters $[5,6]$. One reason might be a strong change in geometry upon ionization resulting in a poor overlap between low vibrational states. This would lead to a heating of the neutralized cluster and perhaps to its dissociation. Although anion photofragmentation might be possible in some cases [11] we do not expect such a strong change in geometry upon neutralization 
(a)

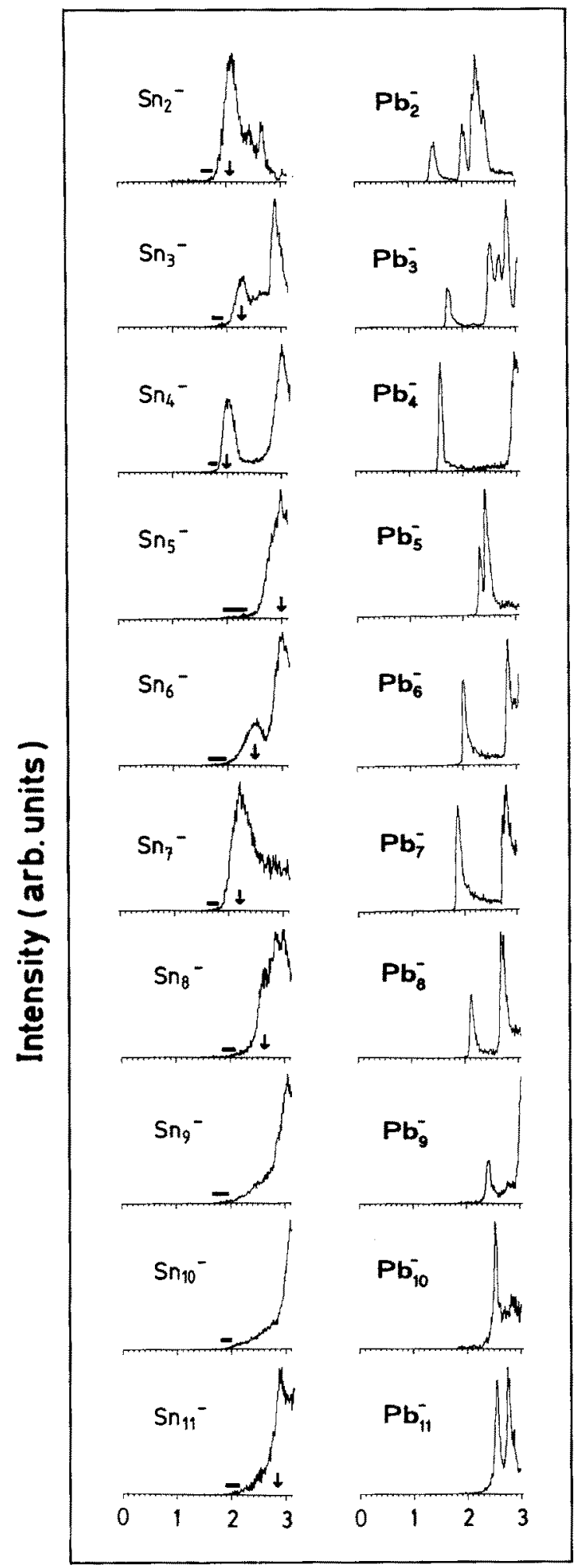

(b)

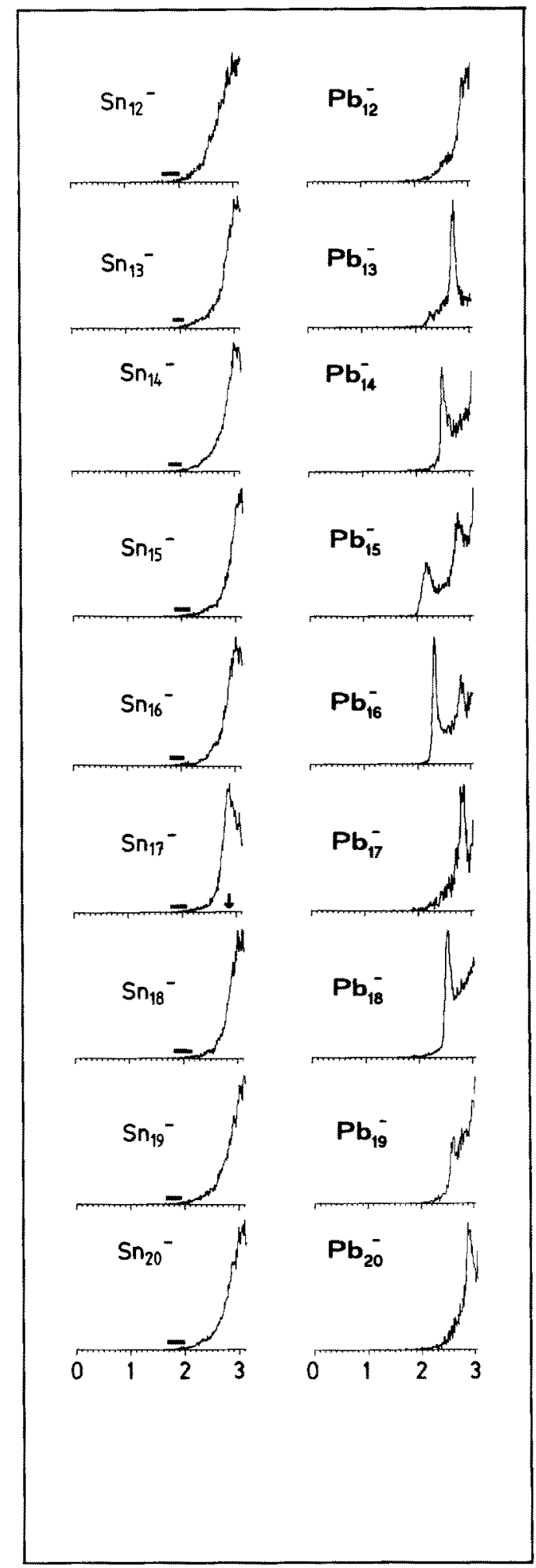

\section{Electron binding energy $[\mathrm{eV}]$}

Fig. 2a and b. Photoelectron energy spectra of tin and lead cluster anions at a detachment energy of $h v=3.68 \mathrm{eV}$. At this photon energy no background correction is necessary. For $\mathrm{Sn}_{n}^{-}$the thresholds are marked by horizontal bars. $\mathrm{Pb}_{n}^{-}$show pronounced peaks near threshold 
Table 1. Photoelectron threshold energies (for $\mathrm{Sn}_{n}^{-}$) and first maximum positions (for $\mathrm{Pb}_{n}^{-}$) as estimates of cluster electron affinities (in $\mathrm{eV}$ )

\begin{tabular}{lcccccccccc}
\hline$n$ & 2 & 3 & 4 & 5 & 6 & 7 & 8 & 9 & 10 & 11 \\
\hline $\mathrm{Sn}_{n}^{-}$threshold & 1.7 & 1.8 & 1.7 & 2.2 & 1.8 & 1.8 & 2.0 & 1.8 & 1.9 & 2.0 \\
$\mathrm{~Pb}_{n}^{-}$1st maximum & 1.45 & 1.70 & 1.55 & 2.35 & 2.05 & 1.95 & 2.15 & 2.45 & 2.55 & 2.55 \\
\hline$n$ & 12 & 13 & 14 & 15 & 16 & 17 & 18 & 19 & 20 & \\
\hline $\mathrm{Sn}_{n}^{-}$threshold & 1.9 & 2.0 & 1.9 & 2.1 & 2.0 & 2.0 & 2.1 & 1.9 & 1.9 & $(2.6)$ \\
$\mathrm{Pb}_{n}^{-}$1st maximum & $(2.6)$ & 2.3 & 2.50 & 2.2 & 2.30 & $(2.85)$ & $(2.55)$ & 2.6 & \\
\hline
\end{tabular}

Table 2. Excitation energies $\mathrm{E}$ of neutral lead cluster in the geometry of the anions, relative to the neutral ground state

\begin{tabular}{lccccccc}
\hline$n$ & 2 & \multicolumn{2}{c}{3} & 4 & 5 & \multicolumn{2}{c}{6} \\
\hline $\mathrm{E}(\mathrm{eV})$ & $0.55,0.80,>1.5$ & $0.80,0.95,1.10, \geqslant 1.30$ & 1.40 & $0.10,>0.70$ & $0.80,>1.0$ \\
$n$ & 7 & 8 & 9 & 10 & 11 & 12 & 13 \\
\hline $\mathrm{E}(\mathrm{eV})$ & $0.90,>1.05$ & $0.60,>0.80$ & $\geqslant 0.60$ & $>0.50$ & $0.20,>0.45$ & $(0.3)$ & $0.4,>0.80$ \\
$n$ & 14 & 15 & 16 & 17 & 18 & 19 & 20 \\
\hline $\mathrm{E}(\mathrm{eV})$ & $\geqslant 0.55$ & $0.60, \geqslant 0.90$ & $0.50,>0.70$ & $(>0.20)$ & $(>0.45)$ & $(\geqslant 0.40)$ & $(>0.15)$ \\
\hline
\end{tabular}

for most of the clusters. Strong isomerization is another possible origin for broad lines and flat thresholds as it had been demonstrated recently with alkali-oxide clusters [12]. Different source conditions should alter the degree of isomerization. Up to now, however, we found no significant change of the PES upon source parameter adjustments.

Comparing the $\mathrm{Sn}_{n}$ data with EDCs of silicon and germanium, $n \leq 12$ [13], we find similar spectra within the range of overlap. The observed photodetachment thresholds of $\mathrm{Si}_{n}^{-}$and $\mathrm{Ge}_{n}^{-}$are essentially the same, whereas the values of $\mathrm{Sn}_{n}^{-}$are shifted to lower binding energies by about $0.5 \mathrm{eV}$ (see Table 1). Only for $n=10$ we find a strong qualitative difference: The $\mathrm{Si}_{10}^{-}$spectrum has a low-energy hump which -according to the jellium model [14-16]-shows that neutral $\mathrm{Si}_{10}$ is a closed shell cluster; in $\mathrm{Ge}_{10}^{-}$such a feature is missing. Also $\mathrm{Sn}_{10}^{-}$(see Fig. 2) has no hump indicating that neutral $\mathrm{Sn}_{10}$ is an open shell cluster, in contrast to $\mathrm{Si}_{10}$.

Photoelectron spectra from lead cluster anions, on the other hand, are different to those of $\mathrm{Si}_{n}^{-}, \mathrm{Ge}_{n}^{-}$, and $\mathrm{Sn}_{n}^{-}$. For most $n$ we find a defined threshold with one or two narrow lines followed by a distinct gap. The energetic positions of neutral excited states (see Table 2 ) vary irregularly with $n$. The existence of a gap indicates that the corresponding neutral cluster is a closed shell species with well separated electronically excited states; in view of this, $\mathrm{Pb}_{n}$ are closed shell clusters for nearly all $n$ observed so far. The narrow lines clearly indicate that isomerization in lead clusters is weak or absent. The gap widths are large and reach $1.4 \mathrm{eV}$ in the case of $\mathrm{Pb}_{4}^{-}$. This is the largest gap we observed so far; it is even larger than that of $\mathrm{Ag}_{8}^{-}[6]$ which-as an alkali-like cluster with nine valence electrons - has one excess electron after a shell closing according to the jellium model. The huge gap for lead at $n=4$ and the low PE-threshold could be interpreted as an $1 p$ shell closing. In this case each $s^{2} p^{2}$-atom gives two electrons to the valence band. A similar behaviour would then also be expected for $n=10$ ( 2 s-closing). The width of the $\mathrm{Pb}_{10}^{-}$band gap cannot be determined as the employed photon energy is too low. The electron affinity, on the other hand, is relatively high, in contrast to the expectation of the jellium model. Instead, $\mathrm{Pb}_{15}^{-}$has a low electron affinity with a pronounced gap indicating that this cluster appears in a special closedshell configuration with high symmetry. As noted earlier, in the mass spectra of $\mathrm{Pb}_{n}^{-}$the line at $n=15$ can be produced with enhanced intensity. We conclude that the simple jellium picture, which has been modelled for monovalent metal clusters, does not work reliably for lead clusters. Instead, it appears to be necessary to consider also the influence of the cluster's geometry.

A complete discussion of the $\mathrm{Sn}_{n}$ and $\mathrm{Pb}_{n}$ photoelectron spectra goes beyond the scope of this communication. In conclusion, we find a strong similarity 
between semiconductor and tin clusters which may be a hint that $\mathrm{Sn}_{n}$ are not free-electron-like species. Bulk electronic bands of group IV diamond structure species (i.e., Si, Ge, Sn) are nearly identical with a decreasing band gap width going from $\mathrm{Si}$ to $\mathrm{Ge}$ and $\mathrm{Sn}$. Bulk lead, on the other hand, is a free-electron-like metal; its photoelectron spectrum reveals two $p$-bands near the Fermi level and an $s$-band at higher binding energies [17]. The cluster photoelectron spectra have to evolve to these bands.

\section{References}

1. Cf. Physics and chemistry of small clusters. NATO ASI Ser. B 158, 755 (1987)

2. Cf. Elemental and molecular clusters. Springer Series in Materials Science, Vol. 6. Berlin, Heidelberg, New York: Springer 1988

3. Leopold, D.G., Ho, J.H., Lineberger, W.C.: J. Chem. Phys. 86, $1715(1987)$

4. Pettiette, C.L., Yang, S.H., Craycraft, M.J., Conceicao, J., Laaksonen, R.T., Cheshnovsky, O., Smalley, R.E.: J. Chem. Phys. 88, $5377(1988)$
5. Ganteför, G., Gausa, M., Meiwes-Broer, K.H., Lutz, H.O.: Z. Phys. D-Atoms, Molecules and Clusters 9, 253 (1988)

6. Ganteför, G., Gausa, M., Meiwes-Broer, K.H., Lutz, H.O.: Faraday Discuss. Chem. Soc. 86, (1989)

7. Kruit, P., Read, F.H.: J. Phys. E 16, 313 (1983)

8. Begemann, W., Dreihöfer, S., Ganteför, G., Siekmann, H.R., Meiwes-Broer, K.H., Lutz, H.O.: Elemental and molecular clusters. Springer Series in Materials Science, Vol. 6, p. 230. Berlin, Heidelberg, New York: Springer 1988

9. Sattler, K., Mühlbach, J., Echt, O., Pfau, P., Recknagel, E.: Phys. Rev. Lett. 47, 160 (1981)

10. LaiHing, K., Wheeler, R.G., Wilson, W.L., Duncan, M.A.: J. Chem. Phys. 87, 3401 (1987)

11. Saunders, W.A., Fayet, P., Wöste, L.: Phys. Rev. A (in press) (1989)

12. Wassermann, B., Martin, T.P.: J. Chem. Phys. (in press)

13. Cheshnovsky, O., Yang, S.H., Pettiette, C.L., Craycraft, M.J., Liu, Y., Smalley, R.E.: Chem. Phys. Lett. 138, 119 (1987)

14. Martins, J.L., Car, R., Buttet, J.: Surf. Sci. 106, 265 (1981)

15. Ekardt, W.: Ber. Bunsenges. Phys. Chem. 88, 289 (1984)

16. Knight, W.D., Clemenger, K., de Heer, W.A., Saunders, W.A., Chou, M.Y., Cohen, M.L.: Phys. Rev. Lett. 52, 2141 (1984)

17. Jezequel, G., Barski, A., Steiner, P.s Solal, F., Roubin, P., Pinchaux, R., Petroff, Y.: Phys. Rev. B 30, 4833 (1984) 